\title{
Adaptable Tool-Path Planning Method for 3D Concrete Printing Based on the Mapping Method
}

\author{
Qian Wan ${ }^{1}$, Li Wang $^{1,2(\bowtie)}$, and Guowei Ma ${ }^{1,2}$ \\ ${ }^{1}$ School of Civil and Transportation Engineering, Hebei University of Technology, 5340 Xiping \\ Road, Beichen District, Tianjin 300401, China \\ wangl1@hebut.edu.cn \\ 2 Tianjin Key Laboratory of Prefabricated Building and Intelligent Construction, Xiping Road, \\ Beichen District, Tianjin 300401, China
}

\begin{abstract}
D concrete printing (3DCP) has been successfully and widely applied in the fields of civil structure, infrastructure, architectural decoration etc.,due to its unique advantages of automation and flexibility, and has shown great potential for development. One of the key steps in the execution of 3DCP is the toolpath planning process. However, 3DCP typically utilize the flowable composite materials which changes with time, and it is easy to induce interface joints or filling defects due to uneven path distribution when constructing the irregularshaped construction. To solve the problem of printing path planning in 3DCP, this paper proposes tool-path planning based on mapping method, which integrates the rheology and necessary continuity of concrete materials into the printing process parameters, improves the mutual adaptability of printing process and material characteristics, improves the continuity of printing, the compactness of filling, and then ensures the stability and durability of printing structure.
\end{abstract}

Keywords: 3D concrete printing (3DCP) - Tool-path planning · Mapping . Adaptable

\section{Introduction}

The $3 \mathrm{D}$ concrete printing (3DCP) method has broken through the traditional construction method of casting components with formworks, so that the irregular shaped and non-standard components can be fabricated easily, the construction design has more freedom, and the structural design for 3DCP constructions can adopt more curvatures. In October 2019, an assembled 3D printing arch bridge with a span of $18.04 \mathrm{~m}$ was built in the campus of Hebei University of technology, Tianjin, China. Although 3DCP is in its preliminary exploration stages, in the construction industry, it shows a rapid development trend $[1,2]$. Tool-path planning is one important step of 3D printing (3DP) execution which is based on the digital model of design calculating the trial of the printhead, usually nozzle mounted on the end-effector of the 3DP device. The current 3DP techniques include contour-parallel method and zigzag method. Although both these 
methods satisfy the tool-path planning of regular cross-section model [3], some problems may occur when doing the irregular shaped construction models tool-path planning in the existent methods.

Tool-path planning is one of the important steps of 3DP technology including 3DCP, which generated the path of the print-head, i.e. the nozzle mounted on the 3D printer's end effector, based on the 3D-digital models. The current tool-path planning methods include the contour-parallel method and the zigzag method. Both of these are competent for the tool-path planning for the model with regular cross-section, e.g. the rectangle or circular sections, which plays an important role in experimental research of materials [3]. However, the current tool-path planning methods will inevitably encounter a lot of problems in respect of 3DCP which is dominated by the irregular shaped components.

The numerous tool-path turnings, tool-path interruptions, and the under fill are common problems of existent 3DCP tool-path planning methods for two main reasons: unreasonable tool-path shape and printing parameters. Contour-parallel tool-path is the offset curve of the model contour, when the size of model is not an integer multiple of the nozzle diameter, there will be a large amount of under fill caused by the self-intersection problem [4, 5]. Zigzag tool-path fills and scans the printing region with a batch of parallel lines, which may cause frequent tool-path turnings and interruptions. The printing parameters include the linear velocity of the print-head during the printing process (printing velocity), and the concrete materials extrusion velocity (extrusion velocity). Due to the fluidity of the concrete materials which change with age, the printing velocity and the extrusion velocity impact the actual width and thickness of materials extrusion. In the recent studies on 3DCP, few objects were studied for the printing parameters, usually using the constant value. However, If the actual extrusion width is inconsistent with the preset, the under fill (or over fill) may appear.

In order to improve the quality of 3DCP components, it is necessary to fully consider the shape of printing in the tool-path planning, to avoid the tool-path interruptions and the under fill, and to reduce the number of tool-path turnings. It's also necessary to evaluate the fluidity of concrete materials to determinate the best printing scheme. In this paper, a novel method of 3DCP tool-path planning method has been proposed, based on the transfinite mapping method and the full consideration of the fluidity. This method can generate tool-path without interruptions, under fill, and frequent tool-path turnings.

\section{Mapping Tool-Path Planning Method}

\subsection{Transfinite Mapping Method}

The transfinite mapping is a method of formulating a surface within the area surrounded by any four curves, the calculation plane is pushed to the physical plane by establishing the mapping function of the natural coordinates $x-y$ and the calculation coordinates $u-v$ $[6,7]$. As shown in Fig. 1, in the physical panel, divides the region A into 4 boundary curves F1, F2, F3 and F4, and each boundary curves corresponds to the 4 edges of the unit square region $F$ on the calculation panel $u$ - $v$. Any point in the calculation panel $u-v$ corresponds to one point in physical panel $x-y$.

Through the printing model is always established by the CAD software, and the printing panel always generated by clipper software, the boundary of printing region 


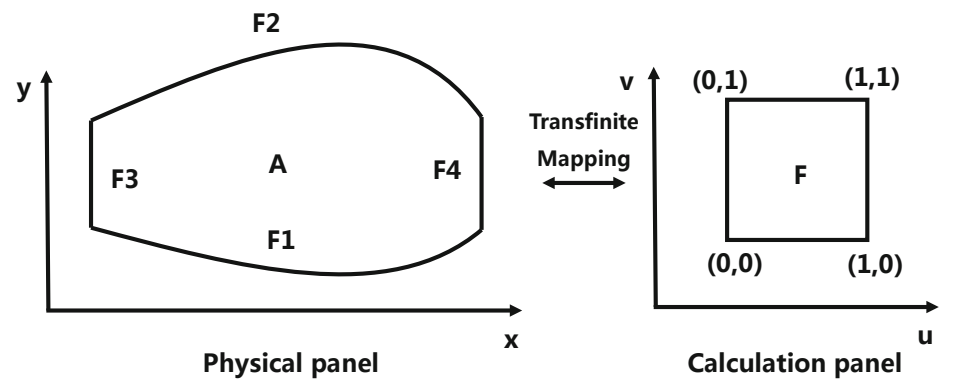

Fig. 1. Physical panel $x-y$ and calculation panel $u-v$

always consists of finite points, shown as polygonal lines. So, in this method, polygonal lines are adopted to approximately describe the shape of the printing region. For a polygonal $f$, assuming the number of nodes is $n$, and the one node can be described with vector as $P_{i}=\left[x_{i}, y_{i}\right]^{T}$. Define the polygonal line length on $P_{i}$ is the length between the $1^{\text {st }}$ node to the $i^{\text {th }}$ node along the polygonal line. For range the polygonal line into [0, 1], the normalization polygonal line length $l_{i}$ on $P_{i}$ is shown in Eq. 1.

$$
l_{i}=\left\{\begin{array}{c}
0 \quad i=1 \\
\frac{\sum_{j=2}^{i}\left|P_{j}-P_{j-1}\right|}{\sum_{j=2}^{n}\left|P_{j}-P_{j-1}\right|} i \neq 1
\end{array}\right.
$$

Using the parametric representation $Q$, a parametric representation of the polygonal line $f$ can be established in Eq. 2 .

$$
p_{i}=\left[x_{i}, y_{i}\right]^{T}=Q\left(l_{i}\right), l_{i} \in[0,1]
$$

For any point in $f$, there is a unique parameter $l$ corresponding to it. For other positions on $\mathrm{f}$ except for nodes, it is determined according to the interpolation from the leading and succeeding nodes, as shown in Eq. 3.

$$
Q(l)=\frac{l-a}{b-a} Q(b)+\frac{b-l}{b-a} Q(a), a<l<b
$$

where: $a(b)$ is the normalization polygonal line length of the closest front(back) node to $Q(l)$.

Using the parametric representation $Q$, the four boundaries F1, F2, F3 and F4 can be formulated as:

$$
\left\{\begin{array}{l}
f_{1 i}=\left[x_{i}, y_{i}\right]^{T}=Q_{1}\left(l_{i}\right) \\
f_{2 i}=\left[x_{i}, y_{i}\right]^{T}=Q_{2}\left(l_{i}\right) \\
f_{3 i}=\left[x_{i}, y_{i}\right]^{T}=Q_{3}\left(l_{i}\right) \\
f_{4 i}=\left[x_{i}, y_{i}\right]^{T}=Q_{4}\left(l_{i}\right)
\end{array}\right.
$$

where $f_{i 1}$ is the $i^{\text {th }}$ node on $\mathrm{F} 1$. 
$v=0$ is one of four boundaries of $F$ on calculation panel $u$ - $v$ corresponding to F1 on $\mathrm{x}-\mathrm{y}$ panel, has only one variable parameter $\mathrm{u}$ and one constant parameter v. So, for F1 and F2, the parameter $l$ can be exchanged with $u$, similarly, for F3 and F4, the parameter $l$ can be exchanged for $v$, as shown in Eq. 5. That is a projector between region $A$ in physical panel $x-y$ and region $F$ in calculation panel $u-v$.

$$
\left\{\begin{array}{l}
f_{1 i}=\left[x_{i}, y_{i}\right]^{T}=Q_{1}(u) \\
f_{2 i}=\left[x_{i}, y_{i}\right]^{T}=Q_{2}(u) \\
f_{3 i}=\left[x_{i}, y_{i}\right]^{T}=Q_{3}(v) \\
f_{4 i}=\left[x_{i}, y_{i}\right]^{T}=Q_{4}(v)
\end{array}\right.
$$
Eq. 6.

The mapping between physical panel $x-y$ and calculation panel $u-v$ is formulated as

$$
\begin{aligned}
P(x, y) & =P_{B}(u, v) \\
& =(1-v) Q_{1}(u)+v Q_{2}(u)+(1-u) Q_{1}(v)+u Q_{2}(v) \\
& -(1-u)(1-v) F(0,0)-(1-u) v F(0,1) \\
& -u v F(1,1)-u(1-v) F(1,0)
\end{aligned}
$$

where $P_{B}(u, v)$ is the mapping function. $F(u, v)$ is the corresponding point of $(u, v)$ in physical panel $\mathrm{x}-\mathrm{y}$, specially, $F(0,0)$ is the $1^{\text {st }}$ point of $\mathrm{F} 1, F(0,1)$ is the $1^{\text {st }}$ point of $\mathrm{F} 2$, $F(1,0)$ is the last point of $\mathrm{F} 1, F(1,1)$ is the last point of $\mathrm{F} 2$.

\subsection{Mapping Tool-Path Planning Method}

Mapping tool-path planning method (MTPPM) is a novel tool-path planning method based on transfinite mapping method. Firstly, dividing the boundary of compensated digital construction model cross-section into 4 curves as F1, F2, F3, and F4. Secondly, establishing the physical panel $\mathrm{x}-\mathrm{y}$ and calculation panel $\mathrm{u}-\mathrm{v}$ and the zigzag liked "module" tool-path. Finally, using mapping function $P_{B}$ to calculate the actual tool-path. The specific steps including: model and parameters input, generation of base-line, establishing of boundary formulations, generation of module tool-path, calculation of actual tool-path, calculation of printing parameters, and NC programs output.

\section{(1) Model and Parameters Input}

In MTPPM, the model is the 2D CAD model of construction, and the smooth curves in model exchanged by polygonal lines, denoted as $\Omega$. The parameters include: the diameter of nozzle $\left(D_{n}\right)$; the number of tool-paths $\left(n_{p}\right)$. The number of tool-paths can be determined by the geometry of model and the diameter of nozzle, specially, can be preliminarily calculated by the ratio of the narrowest dimension of the model $\left(D_{\min }\right)$ to $D_{n}$.

$$
n_{p}=\left\lfloor D_{\min } / D_{n}\right\rfloor
$$




\section{(2) Generation of Base-line}

In 3DP technology, the tool-path is not in contact with the printing region boundary to avoid the over fill. The base-line is the offset line of model boundary, and the distance between model boundary and base-line is $D_{n} / 2$. The base-line denoted as $\Omega$ '.

(3) Establishing of Boundaries Formulations

The base-line $\Omega$ ' was divided into 4 parts, as F1, F2, F3 and F4, based on the interrupt nodes chosen on $\Omega$ '. Thus establishing the mapping function PB following Eq. 1-6. In this method, the interrupt nodes are chosen on UI manually.

(4) Generation of Module Tool-Path

A batch of zigzag tool-path with the number of $n_{p}$ was generated in the unit-square region. The module tool-path can be calculated by Eq. 8, where $p a t h_{i}$ is the $i^{\text {th }}$ module tool-path. The sequence of module tool-path is zigzag-like shape, i.e. if odd module tool-paths $\left(\right.$ path $\left._{\text {odd }}\right)$ are positive sequence, the even module tool-paths $\left(\right.$ path $\left._{\text {even }}\right)$ are inverted sequence. And vice versa.

$$
\operatorname{path}_{i}: v_{i}=\frac{i-1}{n_{p}-1}, u_{i} \in[0,1]
$$

\section{(5) Calculation of Actual Too-Path}

Choosing several nodes on the module tool-path, calculating the actual tool-path following Eq. 6, as shown in Eq. 9.

$$
\operatorname{PATH}_{i}=P_{B}\left(\text { path }_{i}\right)
$$

(6) Calculation of Printing Parameters

Because the distance between each tool-path is variable, the printing parameters are also variable to ensure the complete filling, existing studies [8, 9] have shown that the relationship between printing parameters, printing velocity, extrusion velocity and extrusion width, can be regarded as linear, and can be expressed by Eq. 9 .

$$
v_{f}=\frac{\alpha D_{n} v_{e}}{h D_{f}}
$$

where $v_{f}$ is the printing velocity, $v_{e}$ is the extrusion velocity, $D_{f}$ if the extrusion width, $h$ is the thickness of extrusion, $\alpha$ is an empirical parameter,determined by experiments. 
Different color represents different extrusion width, the maximal width id $1.2 D_{n}$, and the minimal width about is $0.8 D_{n}$. Based on the extrusion graph and Eq. 10, the printing parameters at different position can be determined.

(7) NC Program Output

The NC program always includes the tool-path coordination, and the printing velocity. This study uses Kawasaki robot as a 3D printing device.

\section{Case Study of MTPPM}
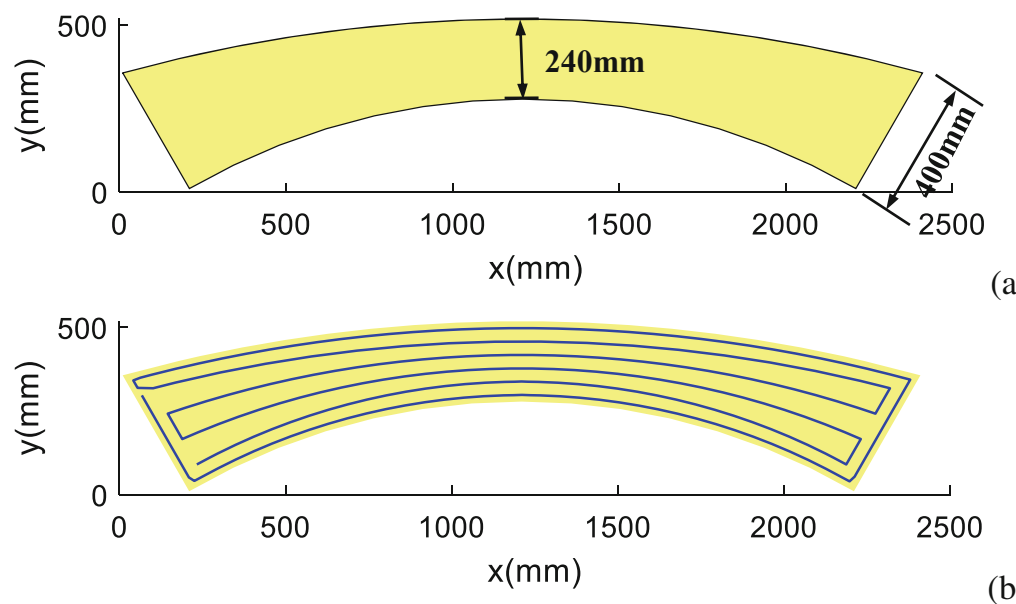

(a)

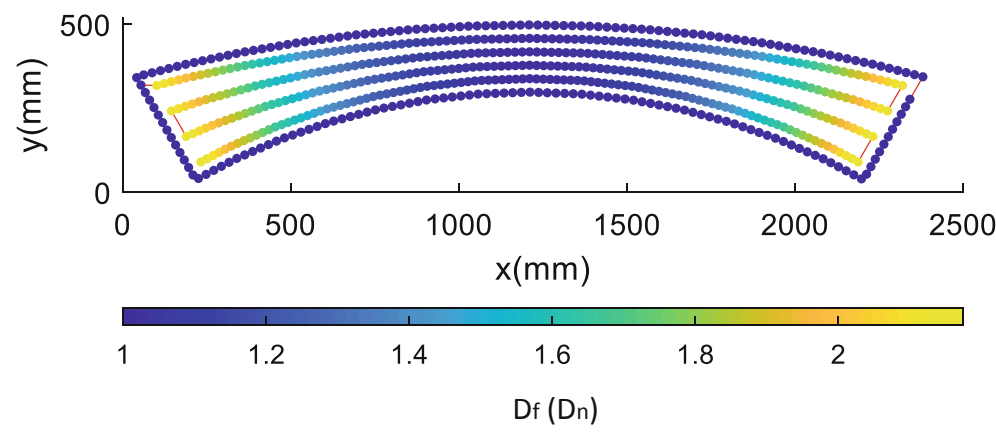

(b)

Fig. 2. Tool-path planning of e variable cross-section shaped arch, (a) the CAD model; (b) the tool-path; (c) extrusion width

In this section, taking the irregular shaped components of the arch bridge construction as an example, the tool-path planning of the three types of variable cross-section 
shaped arch, local irregular shaped arch and multi-component combined shaped arch are discussed respectively, and the feasibility and advantages of the MTPPM are demonstrated.

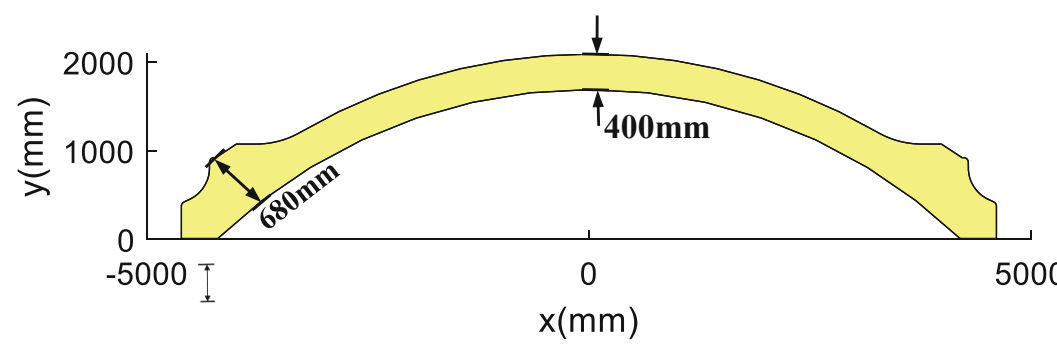

(a)

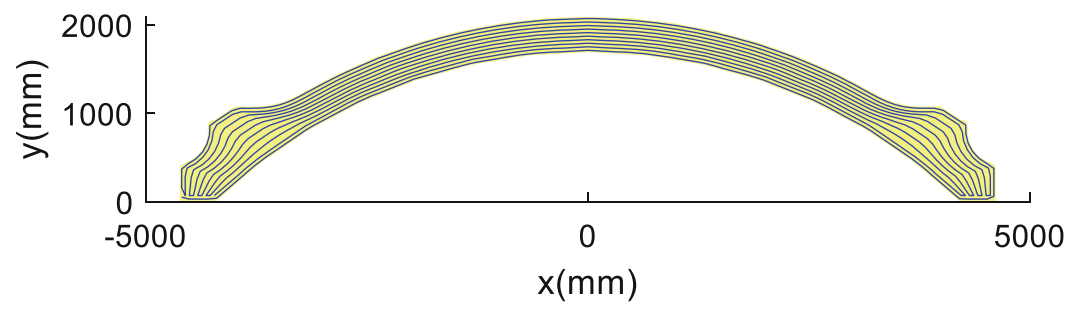

(b)

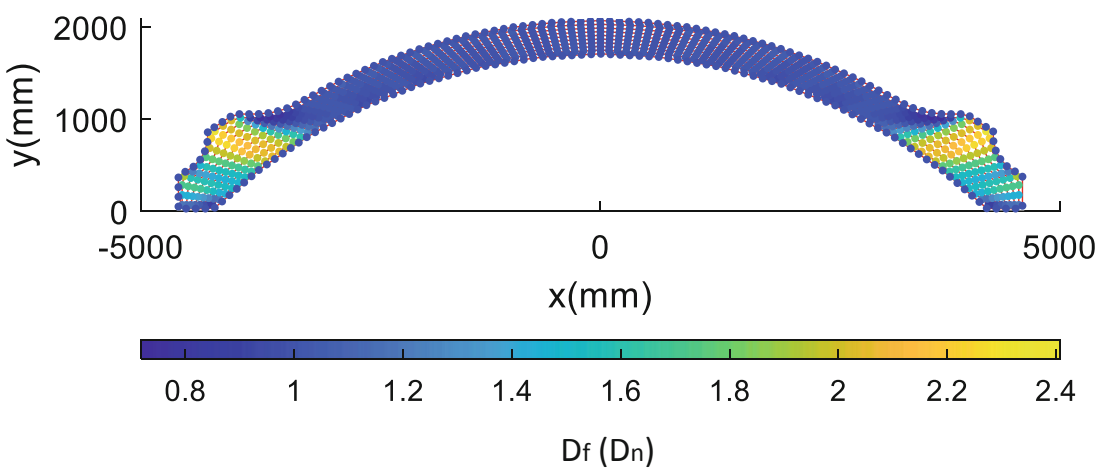

(c)

Fig. 3. Tool-path planning of local irregular shaped arch, (a) the CAD model; (b) the tool-path; (c) extrusion width

\section{Case Study 1: The Variable Cross-section Shaped Arch}

An arch whose cross-section changes gradually from the arch foot to the crown is called a variable-section shaped arch. Using this kind of special-shaped arch can effectively reduce the use of materials and make the structure stress more reasonable. The size of the variable cross-section shaped arch as shown Fig. 2(a), the narrowest length of the arch is $240 \mathrm{~mm}$. To improve the precision of printing, a contour-parallel tool-path set with width of $D_{n}$. If the nozzle diameter is $D_{n}$, the number of expected contour-parallel tool-paths (number of mapping tool-paths) is $240 / 40-2=4$. The printing sequence is internal first, then external. The tool-path as shown in Fig. 2(b), and the extrusion width 
as shown in Fig. 2(c), the maximal extrusion is $2.2 D_{n}$ and the minimal extrusion width is $D_{n}$.

Case Study 2: The Local Irregular Shaped Arch

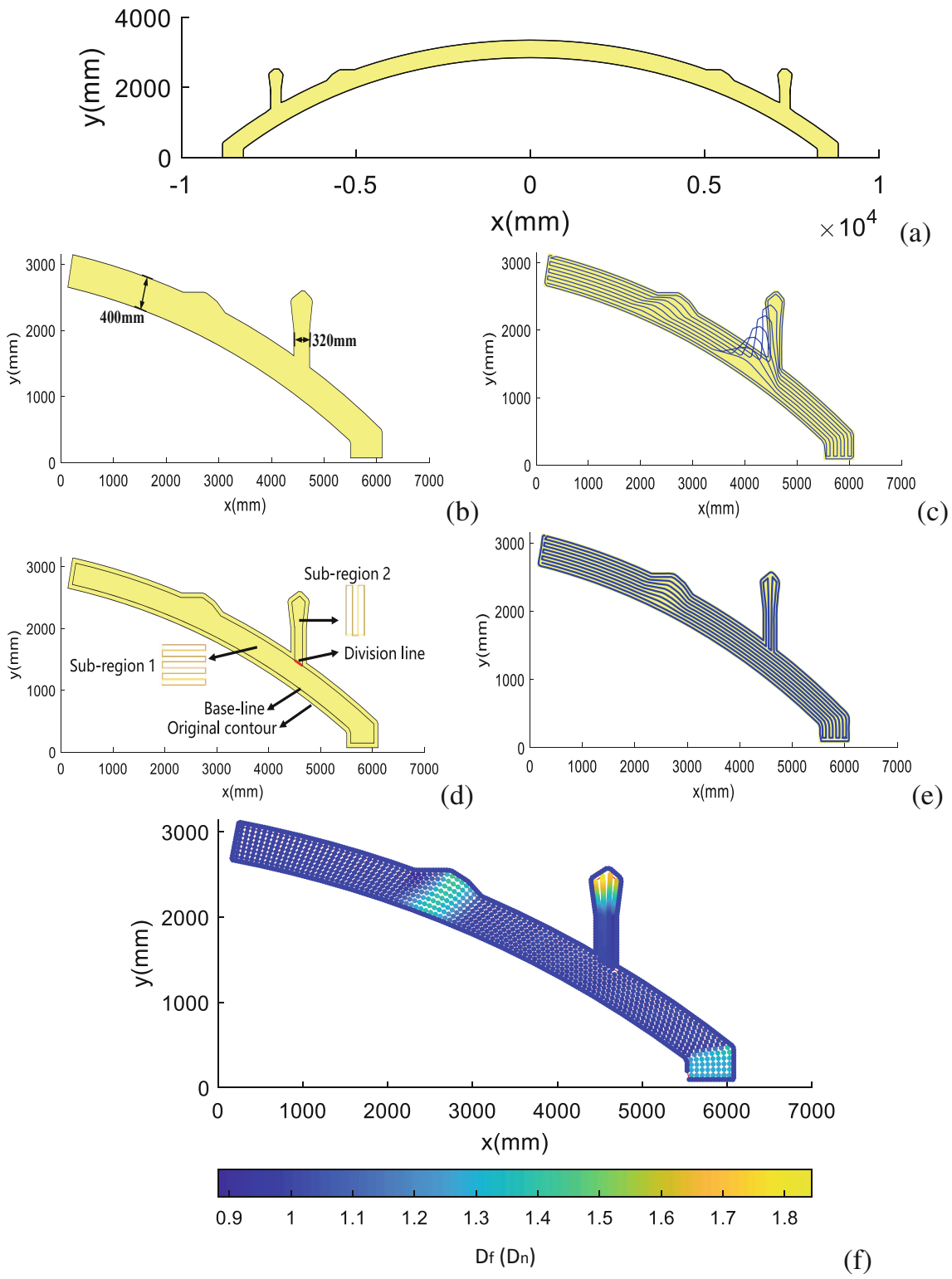

Fig. 4. Tool-path planning of the multi-component combined shaped arch, (a) the CAD model; (b) the right part of model;(c) the "overspill"; (d) division of filling area;(e) the too-path;(f) extrusion width 
As shown in Fig. 3(a), the two ends of the local irregular shaped arch exist additional construction, as the base of other construction, e, g. the column supported the bridge deck. This type of construction using contour-parallel method will lead to large areas of under fill. The specifications of 3DCP using MTPPM is: nozzle diameter is $40 \mathrm{~mm}$, setting one contour-parallel tool-path, the number of the mapping tool-paths is 8 . As shown in Fig. 3(b, c), the most printing with extrusion width of $D_{n}$.

Case Study 3: The Multi-component-combined Shaped Arch

As shown in Fig. 4(a), the construction combining the arch with the columns is called multi-component combined shaped arch. The irregularity of this type of construction is greater than the local irregular shaped arch, and two protruding branches are formed at both ends of the arch. To avoid the "overspill" as shown in Fig. 4(c), the approach of division filling area into several sub-region have been introduced. Taking the right part of the arch shown in Fig. 4(b, d) as an example, the arch ring and the column are divided into two parts, and the tool-path of the arch-region and the column-region are respectively obtained by the MTPPM. The finial tool-path is the continuous combination of the two regions' tool-path.

\section{Conclusion}

The current 3DCP tool-path planning methods: zigzag method and contour-parallel methods inevitably cause tool-path turnings, interruptions and under fill, due the unsuitable tool-path shape and printing parameters, which limit the 3DCP in the actual engineering. This study proposed a novel 3DCP tool-path planning method, based on the transfinite mapping method. Based on the simple module tool-path calculates the extrusion variable tool-path, and adopts variable printing parameters to control the 3DCP process. MTPPM can generate continuous tool-path with no under fill and fewer tool-path turnings. To avoid the "overspill", the division filling region idea has been introduced. The three case studies prove that MTPPM is qualified for the 3DCP process.

\section{References}

1. Panda, B., Tay, Y.W.D., Paul, S.C., Tan, M.J.: Current challenges and future potential of 3D concrete printing. Materialwiss. Werkstofftech. 49(5), 666-673 (2018)

2. Gosselin, C., Duballet, R., Roux, P., Gaudillière, N., Dirrenberger, J., Morel, P.: Large-scale 3D printing of ultra-high performance concrete - a new processing route for architects and builders. Mater. Des. 100, 102-109 (2016)

3. Li, Z., Li, W., Ma, G.: Mechanical improvement of continuous steel microcable reinforced geopolymer composites for 3D printing subjected to different loading conditions. Compos. Part B: Eng. 187(107796), 1-14 (2020)

4. Zhiwei, L., Jianzhong, F., Yong, H., Wenfeng, G.: A robust 2D point-sequence curve offset algorithm with multiple islands for contour-parallel tool path. Comput.-Aided Des. 45, 657-670 (2013)

5. Held, M.: Voronoi diagrams and offset curves of curvilinear polygons. Comput.-Aided Des. 30(4), 287-300 (1998) 
6. Haber, R., Shephard, M.S., Abel, J.F., Gallagher, R.H., Greenberg, D.P.: A general twodimensional, graphical finite element preprocessor utilizing discrete transfinite mappings. Intl. J. Numer. Methods Eng. 17(7), 1015-1044 (1981)

7. de Oliveira Miranda, A.C., Martha, L.F.: Hierarchical template-based quadrilateral mesh generation. Eng. Comput. 33(4), 701-715 (2015). https://doi.org/10.1007/s00366-014-0392-8

8. Tay, Y.W.D., Li, M.Y., Tan, M.J.: Effect of printing parameters in 3D concrete printing: printing region and support structures. J. Mater. Process. Technol. 271, 261-270 (2019)

9. Xu, J., Ding, L., Cai, L., Zhang, L., Luo, H., Qin, W.: Volume-forming 3D concrete printing using a variable-size square nozzle. Autom. Construct. 104, 95-106 (2019)

Open Access This chapter is licensed under the terms of the Creative Commons Attribution 4.0 International License (http://creativecommons.org/licenses/by/4.0/), which permits use, sharing, adaptation, distribution and reproduction in any medium or format, as long as you give appropriate credit to the original author(s) and the source, provide a link to the Creative Commons license and indicate if changes were made.

The images or other third party material in this chapter are included in the chapter's Creative Commons license, unless indicated otherwise in a credit line to the material. If material is not included in the chapter's Creative Commons license and your intended use is not permitted by statutory regulation or exceeds the permitted use, you will need to obtain permission directly from the copyright holder.

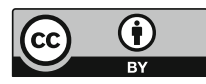

